PART VIII

THEORIES OF LARGE SCALE FIELDS AND THE ACTIVITY CYCLE 


\title{
THEORIES OF LARGE SCALE FIELDS AND \\ THE MAGNETIC ACTIVITY CYCLE
}

\author{
N. O. WEISS \\ Dept. of Applied Mathematics and Theoretical Physics, University of Cambridge, England
}

\begin{abstract}
The magnetic field is effectively frozen into the ionized gas in the Sun and it is therefore necessary first to describe the motion in the convective zone. Large scale motion in giant cells is strongly affected by Coriolis forces, giving a radial shear in the angular velocity, while the interaction of convection and rotation leads to the equatorial acceleration. Many hydromagnetic dynamo mechanisms have been proposed in the last few years. In particular, meridional fields can be generated from azimuthal fields owing to a preferred sense of helicity in the motion $\overline{(\mathbf{u} \cdot \operatorname{curl} \mathbf{u}} \neq 0$, the ' $\alpha$-effect'). Such regeneration is included in Leighton's phenomenological model, which reproduces many features of the solar cycle. More detailed models will have to treat the concentration of magnetic flux into ropes by individual convection cells.
\end{abstract}

\section{Introduction}

It is impossible to provide a comprehensive review in one short lecture and I shall concentrate on a few selected topics in which recent progress has been substantial. Since magnetic fields in the Sun are effectively frozen into the ionized gas it is necessary to describe the motion of this gas before the behaviour of the fields can be understood. Furthermore, the scale of active regions (comparable with the solar radius) indicates that magnetic fields penetrate deep into the convective zone. In the last few years we have gained a better understanding of the structure of this zone and also of the differential rotation, which dominates the solar dynamo. I shall first discuss the velocity field in the convective zone and then go on to recent developments in the theory of hydromagnetic dynamos.

At one time it was reasonable to doubt whether any such dynamo could work: now a host of possible mechanisms has appeared. At the same time, interest has been stimulated by discoveries of recent reversals in the Earth's field and of magnetic fields in stars and pulsars. Some dynamo models have fluid dynamical significance, some relate to the Earth and others imitate the oscillatory behaviour that we see in the Sun. Many details of the solar cycle are reproduced by a pair of remarkably faithful models and the process is becoming more comprehensible. However, these models still describe smoothed mean fields, rather than the complicated patterns that Professor Severny has shown us, and I shall conclude with some remarks about the concentration of magnetic flux into ropes by individual convection cells.

\section{Motion in the Convective Zone}

Models based on mixing length theory ascribe a depth of 100000 to $200000 \mathrm{~km}$ to the convective zone (Baker and Temesvary, 1966). It is conventionally supposed that there is a hierarchy of eddies whose scales are characterised by a mixing length equal 
to the local pressure or density scale height. Unless their vertical size is limited, continuity implies that all flow would be predominantly horizontal. On the other hand, observation shows only a limited number of preferred length scales. The diameter of granules is comparable with the scale height at the base of the photosphere; the formation of sunspots at junctions in the chromospheric network indicates that supergranules are associated with deep-seated convection; Bumba's (1967) suggestion of giant cells is supported by the pattern of magnetic fields (Bumba et al., 1969) and by Howard's (1971) analysis of large-scale velocity fields; and now Bumba (1971) suspects that there may be supergiant cells too. There are some theoretical grounds (Simon and Weiss, 1968) for believing that cells can extend over about three scale heights and therefore that there should, in addition to granules and supergranules, be giant cells with diameters of about $300000 \mathrm{~km}$ and velocities around $0.1 \mathrm{~km} \mathrm{~s} \mathrm{~s}^{-1}$, lasting for periods of a month or more. The convective zone could then be divided into three layers, each with its own preferred scale of motion.

All magnetic features share in the differential rotation of the Sun. Therefore it must be a deep-seated phenomenon and not just a superficial wind. The angular velocity $\Omega$ is found to vary with both latitude and depth. Let us consider the radial variation first. The solar wind exerts a decelerating torque on the convective zone and this deceleration may be enhanced by turbulence (Gough and Lynden-Bell, 1968). It has been suggested (e.g. by Plaskett, 1966) that the Sun's interior rotates ten times faster than the surface, and this hypothesis is supported by measurements of the Sun's oblateness (Dicke, 1970). It is hard to accept the existence of so great a variation in $\Omega$ if any magnetic field permeates the region, and harder still to see how an ambient field could be entirely excluded from the shear zone. If the Sun indeed has a rapidly rotating core it cannot affect magnetic fields observed in the photosphere. Nevertheless, conservation of angular momentum does impose a constraint upon motion in convective cells and the importance of the Coriolis force is given by the ratio of the turnover time to the rotation period of the Sun. For granules this is negligible and for supergranules it is slight. However, for giant cells there will be a tendency to maintain an angular momentum independent of $r$ (the distance from the centre of the Sun) so that $\Omega \propto r^{-2}$; this will be limited by turbulent or kinetic friction. Rising fluid can thus move azimuthally through about $90^{\circ}$ with a consequent distortion of the cells. Recent observations of the Sun's rotation rate (Howard and Harvey, 1970; Wilcox and Howard, 1970) show that magnetic features rotate more rapidly than the surrounding photosphere and also that the large scale field has a shorter rotation period than sunspots, thereby supporting the hypothesis that $\partial \Omega / \partial r<0$.

It is apparent that the observed equatorial acceleration must result from the effect of rotation upon convection in a spherical shell and various simplified models have been put forward to explain this. Plaskett (1959) suggested that a heliostrophic wind could be driven by the meridional pressure gradient resulting from cooling at the poles, where rotation inhibits convection. (It is only in axisymmetric systems that convection is suppressed at the equator (Durney, 1968, 1970).) If convection cells near the equator are elongated parallel to the rotation axis, the temperature difference on an 
equipotential surface might be $30^{\circ}$, which would suffice to drive the differential rotation (Weiss, 1965). However, there is no direct evidence for such elongation and the observations summarized by Caccin et al. (1970) indicate that the temperature difference is less than $20^{\circ}$, while Plaskett $(1962,1970)$ used measurements of limb darkening to find an excess temperature of $300^{\circ}$ at the poles.

Biermann (1958) introduced an isotropic eddy viscosity to describe the transport of angular momentum by convective eddies; reduced diffusion in the radial direction then leads to a meridional circulation with equatorward motion at the surface. That such a motion can produce an equatorial acceleration follows from a simple argument due to Kippenhahn (1964). Let $(r, \theta, \varphi)$ be spherical polar co-ordinates and consider the region $r_{1}<r<r_{0}$ of a system rotating with constant angular velocity $\Omega$. Suppose there is an axisymmetric meridional flow with a $\theta$-component $v(r, \theta)$ such that $v<0$ $\left(r_{1}<r<r_{2}\right)$ and $v>0\left(r_{2}<r<r_{0}\right)$. Then conservation of mass implies that

$$
\int_{r_{1}}^{r_{2}} \varrho v r \mathrm{~d} r=\mid \int_{r_{2}}^{r_{0}} \varrho v r \mathrm{~d} r .
$$

Hence, comparing the transport of angular momentum in the two regions, we find that

$$
\int_{r_{1}}^{r_{2}} \varrho v \Omega r^{3} \mathrm{~d} r|<| \int_{r_{2}}^{r_{0}} \varrho v \Omega r^{3} \mathrm{~d} r \mid .
$$

Thus angular momentum is transported towards the equator and the flow can maintain a steady state with an equatorial acceleration. This behaviour has been demonstrated in specific models (Kippenhahn, 1963; Köhler, 1966) and Köhler (1970) has shown that anisotropic viscosity leads to an equatorial acceleration for an incompressible fluid in a spherical shell, constrained to rotate between free boundaries.

Another approach draws on meteorological analogy. In the terrestrial atmosphere transport of angular momentum is dominated by baroclinic (Rossby) waves. These waves have been studied theoretically and in laboratory experiments, using a rapidly rotating cylindrical annulus with an imposed lateral temperature gradient (Hide, 1970). A symmetrical regime is unstable and growing waves develop into asymmetric eddies. The flow is predominantly horizontal, and is dominated by the Coriolis force and horizontal pressure gradients; any unstable vertical variation in temperature must be small compared with the horizontal temperature difference. This baroclinic instability gives rise to cyclones or anticyclones in the Earth's atmosphere. Attempts have been made to identify baroclinic waves in the Sun, either from the proper motions of sunspots (Ward, 1965, 1966) or by directly measuring velocities (Plaskett, 1966), and then to show that these waves are responsible for the equatorial acceleration (Starr and Gilman, 1965; Kato,1969; Kato and Nakagawa, 1969; Starr, 1968, has conveniently assembled most of the relevant papers). The essential feature of this process is that the acceleration is maintained not by a general meridional circulation but owing to a net non-linear transfer of angular momentum by non-axisymmetric eddies. The discussion 
of this effect is most illuminating but the model cannot be applied directly to the Sun. The convective zone differs significantly from the Earth's atmosphere. The superadiabatic stratification cannot be neglected, for the transport of energy requires large scale vertical motions in regions where the scale height is an appreciable fraction of the solar radius. Observationally, Rossby waves cannot be distinguished from the giant cells described above and horizontal motions should not be treated independently of convection.

These simplified models have demonstrated that an equatorial acceleration is less remarkable than had once been supposed. Further progress requires a proper study of non-linear convection in rotating systems. Busse (1970) has formulated the problem for convection in a Boussinesq fluid contained in a slowly rotating spherical shell. Different modes are described by spherical harmonics in the form

$$
f(r) P_{l}^{m}(\cos \theta) e^{i m \varphi} .
$$

The first mode to become unstable has $m=l$, corresponding to a sectorial harmonic with a segmented structure like an orange and a pronounced maximum at the equator. For a thin shell of depth $h$, instability sets in with

$$
l \approx \frac{\pi r_{0}}{\sqrt{2 h}}
$$

thus motion occurs in rolls whose equatorial cross-section is rectangular with an aspect ratio of $\sqrt{ } 2$. Proceeding to higher order in an expansion about the critical Rayleigh number, Busse shows that there is a net equatorial acceleration whose magnitude (after inserting suitable values for parameters) is comparable with that observed in the Sun. Similar results were obtained by Davies-Jones and Gilman (1970, 1971). As a model for giant cells they considered a thin cylindrical annulus with rectangular cross-section, rotating about a vertical axis and heated from below. The relevant equations were solved to second order. When rotation is dominant the motion tends to be in rolls and produces differential rotation with an equatorial acceleration. Durney $(1970,1971)$ has used a computer to tackle a more complicated model: the problem resembles that of Busse, with free boundaries, but non-linear terms are represented in the mean field (Herring) approximation. This provides (as is necessary) a limited representation of non-axisymmetric flow, though there is no coupling between different values of $m$. The fields are expanded in vector spherical harmonics and truncated; only three poloidal and two toroidal harmonics, each with five radial functions, are retained. The qualitative features of Busse's analysis are confirmed by Durney's results. The dominant mode has the predicted value of $l$ and the heat flux is maximized when $m=l$; the estimated effect of fluctuating interactions is to mix angular momentum and to produce an equatorial acceleration; the heat transport is a maximum at the equator (though the inhibition of convection at the poles is enhanced by the Herring approximation); and the convection pattern lags behind the rotation of the shell as a whole. 
Obviously further work remains to be done but it is now possible to give a plausible model of the convective zone. The radiative core rotates with a constant angular velocity. In the convective zone, below about $15000 \mathrm{~km}$ convection occurs in giant (or possibly in supergiant) cells and $\partial \Omega / \partial r<0$. These cells are probably elongated perpendicular to the equator. Above this level are the supergranular cells in which the horizontal velocity is predominantly outward from a rising central plume. The Coriolis force therefore introduces a swirling flow in the opposite sense to the Sun's rotation (clockwise in the northern hemisphere, anti-clockwise in the southern, like terrestrial anticyclones) corresponding to a net radial vorticity. An equatorial acceleration is present throughout but drops to zero at the base of the convection zone. We might speculate that the existence of longitudinal structure in the field (Bumba et al., 1969) and of magnetic sectors with a fixed rotation period of 27 days (Wilcox and Ness, 1967) indicates the presence of convection in rolls parallel to the axis of rotation. However, preferred longitudes for solar activity might persist as a consequence of the dynamo process itself (Leighton, 1969) in which case it can only be inferred that the 27 day rotation period is somehow typical of the deep convection zone.

\section{The Dynamo Problem and Models of the Solar Cycle}

Despite the presence of strong local fields, the average magnetic energy density is small compared with the energy of motion. It is naturally convenient to consider smoothed magnetic fields and to treat them kinematically. The main features of the dynamo that produces the solar cycle are now generally accepted (Babcock, 1961; Leighton, 1964; Schmidt, 1968). The process runs as follows:

(i) At sunspot minimum there is an initial poloidal field and the toroidal field is small.

(ii) Differential rotation forms a strong toroidal field, first at medium and then at lower latitudes.

(iii) Some instability produces kinks in the toroidal field, which float upwards (or are borne up by rising supergranules) to the surface. The associated swirl produces tilted bipolar regions with the preceding part closer to the equator, thus generating a poloidal field opposite to that originally present.

(iv) The supergranules provide an effective eddy diffusion, which may be represented by a random walk process, and the reversed poloidal field diffuses to the poles by the next sunspot minimum.

(v) The sequence is then repeated to give the full cycle.

More detailed investigation involves the general theory of hydromagnetic dynamos. Fifteen years ago there was doubt as to whether any homogeneous dynamo mechanism was possible (Cowling, 1953). This gloom has been dispelled: indeed, G. O. Roberts (1970) has recently stated that 'almost all motions' will give dynamo action. (The phrase is given a mathematically precise meaning and obvious symmetrical models are still excluded.) Thus Lortz (1968) has devised a self-exciting dynamo with helical symmetry, while G. O. Roberts (1971), starting with an axially symmetric velocity, has 
produced a growing, non-axisymmetric field. The present state of dynamo theory is reviewed in detail by $\mathbf{P}$. H. Roberts (1971) and discussed by Parker (1970a, b) in the context of astronomy.

A successful dynamo must avoid the effects of Cowling's theorem. The induction equation has the form

$$
\frac{\partial \mathbf{B}}{\partial t}=\operatorname{curl}(\mathbf{u} \times \mathbf{B})+\eta \nabla^{2} \mathbf{B},
$$

where $\mathbf{B}$ is the magnetic field, $\mathbf{u}$ is the velocity and $\eta$ the magnetic diffusivity. Suppose that $\mathbf{B}$ and $\mathbf{u}$ are axisymmetric. They can be separated into poloidal (meridional) and toroidal (azimuthal) parts:

$$
\mathbf{B}=\mathbf{B}_{p}+\mathbf{B}_{\varphi} \mathbf{i}_{\varphi}, \quad \mathbf{u}=\mathbf{u}_{p}+\mathbf{u}_{\varphi} \mathbf{i}_{\varphi} .
$$

It is convenient to introduce as an independent variable the distance from the axis,

$$
\varpi=r \sin \theta
$$

together with the operator

$$
\Delta=\nabla^{2}-\frac{1}{\varpi^{2}}
$$

Then the $\varphi$-component of (1) becomes

$$
\frac{\partial}{\partial t}\left(\frac{B_{\varphi}}{\varpi}\right)+\nabla \cdot\left(\frac{B_{\varphi}}{\varpi} \mathbf{u}_{p}\right)=\mathbf{B}_{p} \times \nabla\left(\frac{u_{\varphi}}{\varpi}\right)+{ }_{\varpi}^{1} \eta \Delta B_{\varphi} .
$$

In this equation the second term on the left hand side merely represents the advection of toroidal field by the flow. On the right hand side, the creation of toroidal flux from the poloidal field as a result of differential rotation compensates for ohmic dissipation. The poloidal field is best expressed in terms of a toroidal vector potential,

$$
\mathbf{B}_{p}=\operatorname{curl}\left(A \mathbf{i}_{\varphi}\right) .
$$

Then (1) can be integrated to give

$$
\frac{\partial}{\partial t}(\varpi A)+\mathbf{u}_{p} \cdot \nabla(\varpi A)=\eta \varpi \Delta A .
$$

In contrast with (2), Equation (3) does not allow the regeneration of poloidal flux from $B_{\varphi}$. Consequently $\mathbf{B}_{p}$ ultimately decays, followed by $B_{\varphi}$. Thus an axisymmetric field cannot be maintained (Cowling, 1934).

A realistic dynamo model must therefore be non-axisymmetric, with an azimuthally averaged field $\mathbf{B}$, whose poloidal part is maintained by a toroidal emf

$$
E_{\varphi}=\overline{(\mathbf{u} \times \mathbf{B})_{\varphi}} \text {. }
$$


Most models incorporate two scales of motion. Averaging over $\varphi$ then produces a simplified form of $E_{\varphi}$ which can be used to solve (1) for the mean field B. Parker $(1955,1970 \mathrm{~b})$ argued heuristically that the rate of regeneration of the poloidal field in the Sun as flux tubes are brought up to the surface should be proportional to the toroidal field strength, so that

$$
E_{\varphi}=\Gamma B_{\varphi}
$$

and (3) becomes

$$
\frac{\partial A}{\partial t}+\frac{1}{\varpi} \mathbf{u}_{p} \cdot \nabla(\varpi A)=\Gamma B_{\varphi}+\eta \Delta A .
$$

Dynamo action is then possible. For example, if deviations from axial symmetry are small and the magnetic Reynolds number $R_{m} \gg 1$, it is possible to expand $\mathbf{B}$ and $\mathbf{u}$ in powers of $R_{m}^{-1 / 2}$. Then (2) and (5) hold for modified average fields $B_{\varphi}$ and $A$, with $\Gamma$ a function of $\mathbf{u}_{p}$, and dynamo action can be demonstrated (Braginsky, 1964).

In order to maintain the magnetic field it is necessary that the system should not be axially symmetric and this is certainly true of the turbulent convective zone. But "order does not arise spontaneously out of chaos" (Cowling, 1965). For homogeneous isotropic turbulence the average quantities $\overline{\mathbf{u}}, \overline{\operatorname{curl} \mathbf{u}}$ and $\overline{\mathbf{u} \cdot \operatorname{curl} \mathbf{u}}$ are all zero. However, if the random turbulence lacks reflectional symmetry it will have a preferred sense of helicity, so that

$$
\overline{\mathbf{u} \cdot \operatorname{curl} \mathbf{u}} \neq 0 \text {. }
$$

The effect of this small scale turbulent flow on the mean field $\mathbf{B}$ is given by a mean emf

$$
E=\alpha \mathbf{B}-\beta \operatorname{curl} \mathbf{B}
$$

with

and

$$
\alpha=-\frac{1}{3} \tau \overline{\mathbf{u} \cdot \operatorname{curl} \mathbf{u}}
$$

$$
\beta=\frac{1}{3} \tau \overline{\mathbf{u} \cdot \mathbf{u}}
$$

where $\tau$ is the correlation time for a turbulent eddy. Then (5) is once more obtained in the form

$$
\frac{\partial A}{\partial t}+\frac{1}{\varpi} \mathbf{u} \cdot \nabla(\varpi A)=\alpha B_{\varphi}+(\eta+\beta) \Delta A .
$$

Thus the helicity allows regeneration of the poloidal field by the so-called ' $\alpha$-effect' in (7). That such a dynamo operates has been shown by the Potsdam group (Steenbeck et al., 1966; Steenbeck and Krause, 1966, 1969; Krause et al., 1971) on the assumption that

$$
v^{2} \tau \ll l^{2} / \tau \ll \eta
$$

(where $v, l$ are typical velocities and length scales for the turbulent eddies) and also by 
Moffatt (1970) on the assumption that $v l / \eta \ll 1$. As indicated below, this theory is also the basis for a model of the solar cycle.

The various models of the sun represent smoothed fields and include some form of eddy diffusion. The effect of a meridional circulation on a poloidal field has been computed by Maheswaran (1969), while Nakagawa and Swarztrauber (1969) have studied the generation of toroidal flux. They adopt an axisymmetric velocity that satisfies the equation of motion for an incompressible fluid in a spherical shell with boundary conditions such that $\partial \Omega / \partial r>0$. Differential rotation creates $B_{\varphi}$ from $\mathbf{B}_{p}$ in a zone that migrates equatorward with velocity $\mathbf{u}_{p}$ (Bullard, 1955) but more complicated flows are needed to reverse $\mathbf{B}_{p}$ (Nakagawa, 1971).

Many features of the solar cycle are reproduced when the field is distorted by baroclinic waves and Gilman $(1968,1969 a, b)$ has argued that the Sun is indeed a Rossby wave dynamo. He considers a simplified model with waves driven by horizontal temperature gradients in a small, rapidly rotating cylindrical annulus. The fields are represented by drastically truncated expansions but the Coriolis force does allow generation of reversed poloidal flux from the toroidal field, leading to an oscillatory dynamo. A more realistic model, with spherical symmetry, has been studied by Gordon (1970). Similar processes can be expected in the convective zone, where the superadiabatic vertical gradient is more important than temperature differences between the poles and the equator (Davies-Jones and Gilman, 1970).

A successful model has been constructed in which the interaction between convection and rotation is represented by the $\alpha$-effect (Steenbeck and Krause, 1969; Krause et al., 1971). Owing to the density stratification, rising material is associated with an outward radial flow and thus with anticyclinic motion. Thus turbulent convection has a left-handed screw sense in the northern hemisphere, and righthanded in the southern. An expression for $\alpha$ emerges after solving the NavierStokes equation and Krause (1968) finds that (6) reduces to

$$
\alpha=\frac{v^{2} \tau^{2} \Omega}{H} \cos \theta,
$$

where $H$ is the density scale height. The resistivity is dominated by the eddy diffusivity $\beta$. The model separates the convective zone into a lower region (giant cells) with differential rotation such that $\partial \Omega / \partial r<0$, and an upper region (supergranules) in which the $\alpha$-mechanism operates. With suitably chosen parameters reversals are obtained and a convincing butterfly diagram is produced.

This turbulent dynamo resembles Leighton's (1969) physical model, which reproduces the solar cycle with extraordinary accuracy. In this model the fields are projected onto a sphere of radius $r_{0}$ (by averaging radially through the convective zone) and then averaged over longitude to give mean fields that are functions only of the colatitude and time. The differential equations governing $\mathbf{B}$ are constructed from terms representing the physical effects responsible for the solar cycle. Once again, $\Omega$ is assumed to vary in the lower convective zone. In the upper region, toroidal fields erupt and form poloidal fields which are in turn dispersed by a random walk process. 
Furthermore, the eruption occurs only when $\left|B_{\varphi}\right|$ exceeds a critical value $B_{c}$. The essential equations are then

$$
\frac{\partial B_{r}}{\partial t}=F \delta \frac{C}{\sin \theta} \frac{\partial}{\partial \theta}\left(B_{\varphi} \sin \gamma\right)+\frac{1}{T_{D} \sin \theta} \frac{\partial}{\partial \theta}\left(\sin \theta \frac{\partial B_{r}}{\partial \theta}\right)
$$

and, from (2),

where

$$
\frac{\partial B_{\varphi}}{\partial t}=\sin \theta\left(B_{\theta} \frac{\partial \Omega}{\partial \theta j}+r_{0} B_{r} \frac{\partial \Omega}{\partial r}\right)-\delta \cdot C^{\prime}|B \varphi| B_{\varphi},
$$

$$
\begin{array}{rlrl}
\delta & =0 & & \left(\left|B_{\varphi}\right|<B_{c}\right) \\
& =1 & \left(\left|B_{\varphi}\right| \geqslant B_{c}\right)
\end{array}
$$

and $C, C^{\prime}$ are constants depending on properties of the convective zone. The remaining field component, $B_{\theta}$, is calculated from $\nabla \mathbf{B}=0$. In (9), the first term on the right-hand side generates new poloidal flux. The arbitrary parameter $F$ is an adjustable efficiency factor and $\gamma$ is the tilt of active regions. Now this term corresponds to an emf.

with

$$
E_{\varphi}=\Gamma B_{\varphi}
$$

$$
\Gamma=F \delta \frac{C \sin \gamma}{\sin \theta}
$$

cf. Equation (8), and Leighton takes $\sin \gamma=\frac{1}{2} \cos \theta$. Thus his mechanism is similar to the $\alpha$-effect described above. ( $\Gamma$ remains finite since $\left|B_{\varphi}\right|<B_{c}$ when $\theta$ is small.) The remaining term in (9) represents the random walk process, with a decay time $T_{D} \approx 22 y$, corresponding to the eddy diffusivity in (7). Similarly, Equation (10), which includes the effects of differential rotation in the lower region and depletion of $B_{\varphi}$ as a consequence of eruption into the upper zone, resembles (2). The main features of the solar cycle - Spörer's law, the butterfly diagram, the poleward drift of prominence zones and the 22 year periodicity - can be reproduced for a variety of assumptions about $\Omega(r, \theta)$. In this paper, Leighton discusses a number of experiments in which the values of parameters are altered. For example, the dynamo is found to be most efficient (i.e. the value of $F$ that allows a 22 yr period is least, about 0.6 ) when the differential rotation is dominated by a radial variation such that

$$
h \frac{\partial \Omega}{\partial r}=-18 \sin ^{2} \theta \text { radians } y^{-1} .
$$

This heuristic model demonstrates that the observed behaviour of the Sun's magnetic field is compatible with the dynamo process outlined at the beginning of this section. Work by others, notably the Potsdam group, shows that the model can be made more rigorous. Many details of these mean field dynamos remain to be investigated. In addition it is now necessary to seek a better understanding of the effect of individual convection cells in producing the flux ropes that emerge into the photosphere. 


\section{Concentration of Magnetic Fields into Flux Ropes}

Magnetic flux is mainly concentrated around the perimeter of solar convection cells and particularly at corners where several cells meet. For weak fields this concentration is purely kinematic and the field is limited by the magnetic Reynolds number

$$
R_{m}=\frac{v l}{\eta} .
$$

A steady solution of (1) with a balance between diffusion and advection is achieved after the initial field $\mathbf{B}_{0}$ has been amplified locally to a magnitude

$$
B \approx R_{m}^{1 / 2} B_{0} \quad \text { or } \quad B \approx R_{m} B_{0}
$$

for two or three dimensional flow respectively (Parker, 1963; Clark, 1965; Weiss, 1966; Clark and Johnson, 1967). In the Sun $R_{m}$ is so large that forces exerted by these fields could not be withstood. It is generally supposed that concentration proceeds until equipartition is achieved. Then the field is limited by dynamical effects, and, locally,

$$
\frac{1}{2} \varrho u^{2} \approx \frac{B^{2}}{2 \mu} .
$$

Indeed, Beckers (1971) has listed this as a means of determining the field strength. However, there has hitherto been no more precise calculation on which this assumption could be based.

The process can be studied through a simple model problem. Consider a layer of incompressible fluid, heated from below in the presence of an imposed vertical field $\mathbf{B}_{0}$. The linearised treatment is well understood (Chandrasekhar, 1961; Danielson, 1961 ; Weiss, 1964) and non-linear solutions have recently been obtained on a computer. Three regimes are found to exist, depending on the value of $B_{0}$ : weak fields are distorted kinematically and reach a maximum strength given by (12), moderate fields are concentrated until they are powerful enough to affect the motion, while strong fields hinder and ultimately suppress convection, as predicted by linear theory. Preliminary results for two dimensional flow within free boundaries show a maximum magnetic energy density for moderate fields that is four to ten times greater than the kinetic energy density corresponding to the greatest horizontal velocity. The strongest fields may therefore be several times greater than the values derived from (13). Moreover, the computations confirm not only that overstable linear modes grow into finite amplitude oscillations but also that certain exponentially growing perturbations develop into non-linear oscillations.

Further investigation should help to clarify the factors governing the formation of flux ropes and also, perhaps, to explain the transmission of energy in sunspots (Weiss, 1969). The magnetic field in sunspot umbrae is strong enough to inhibit steady convection, yet the energy emitted cannot be supplied by radiative transfer. As 
Sweet $(1971)$ and Wilson $(1969,1971)$ have already pointed out, some wave process must be present. Savage (1969) considers that overstable modes can be excited in sunspots, owing to a coupling of hydromagnetic waves in the unstable region with gravity waves in the stably stratified layer above.

\section{Conclusion}

It is apparent that great advances have been made in our understanding of large scale magnetic fields in the Sun. Many suggestive models illuminate various aspects of the solar cycle; but details are frequently obscure and more comprehensive calculations have still to be completed. A proper treatment of the convective zone must include the effects of compressibility as well as rotation, in three dimensional spherical geometry. The development of photospheric fields by flux concentration between individual granules and supergranules must be studied, as proposed by Kuklin (1971). And these results must be combined with the full dynamo problem in order to provide a proper model that can explain the complicated fields that Severny (1971) has described.

\section{References}

Ambrož, P., Bumba, V., Howard, R., and Sýkora, J.: 1971, this volume, p. 696.

Babcock, H. W.: 1961, Astrophys. J. 133, 572.

Baker, N. and Temesvary, S.: 1966, Tables of Convective Stellar Envelope Models, NASA, New York. Beckers, J. M.: 1971, this volume, p. 3.

Biermann, L.: 1958, in Electromagnetic Phenomena in Cosmical Physics, (ed. by B. Lehnert), Cambridge University Press.

Braginsky, S. I.: 1964, Zh. Eks. Teor. Fiz. 47, 1084 Soviet Phys. JETP 20, 726, 1965.

Bullard, E. C.: 1955, in Vistas in Astronomy 1 (ed. by A. Beer), Pergamon, London.

Bumba, V.: 1967, in Plasma Physics (ed. by P. A. Sturrock), Academic Press, London.

Bumba, V., Howard, R., Kopecky, M., and Kuklin, G. V.: 1969, Bull. Astron. Inst. Czech. $20,18$.

Busse, F. H.: 1970, Astrophys. J. 159, 629.

Caccin, B., Falciani, R., Moschi, G., and Rigutti, M.: 1970, Solar Phys. 13, 33.

Chandrasekhar, S.: 1961, Hydrodynamic and Hydromagnetic Stability, Clarendon Press, Oxford.

Clark, A., Jr.: 1965, Phys. Fluids 7, 1455.

Clark, A., Jr. and Johnson, A. C.: 1967, Solar Phys. $2,433$.

Cowling, T. G.: 1934, Monthly Notices Roy. Astron. Soc. 94, 39.

Cowling, T. G.: 1953, in The Sun (ed. by G. R. Kuiper), University of Chicago Press.

Cowling, T. G.: 1965, in Solar and Stellar Magnetic Fields (ed. by R. Lüst), North-Holland, Amsterdam.

Danielson, R. E.: 1961, Astrophys. J. 134, 289.

Davies-Jones, R. P. and Gilman, P. A.: 1970, Solar Phys. $12,3$.

Davies-Jones, R. P. and Gilman, P. A.: 1971, J. Fluid Mech., to be published.

Dicke, R. H.: 1970, Astrophys. J. 159, 1.

Durney, B.: 1968, J. Atmospheric Sci. 25, 771.

Durney, B.: 1970, Astrophys. J. $161,1115$.

Durney, B.: 1971, Astrophys. J. 163, 353.

Gilman, P. A.: 1968, Science 160, 760.

Gilman, P. A.: 1969a, Solar Phys. 8. 316.

Gilman, P. A.: 1969b, Solar Phys. 9, 3.

Gordon, C. A.: 1970, Ph.D. thesis, Mass. Inst. Tech.

Gough, D. O. and Lynden-Bell, D.: 1968, J. Fluid Mech. 32, 437. 
Hide, R.: 1970, in Global Circulation of the Atmosphere (ed. by G. A. Corby), Roy. Meteorol. Soc., London.

Howard, R.; 1971, Solar Phys. 16, 21.

Howard, R. and Harvey, J.: 1970, Solar Phys. 12, 23.

Kato, S.: 1969, Astrophys. J. 157, 827.

Kato, S. and Nakagawa, Y.: 1969, Solar Phys. 10, 476.

Kippenhahn, R.: 1963, Astrophys. J. 137, 664.

Kippenhahn, R.: 1964, in Atti del convegno sulli campi magnetici solari e la spettroscopia ad alta risoluzione (ed. by M. Cimino), Barbera, Firenze.

Köhler, H.: 1966, Mitt. Astron. Ges. 21, 91.

Köhler, H.: 1970, Solar Phys. 13, 3.

Krause, F.: 1968, Habilitationsschrift, Universität Jena.

Krause, F. and Rädler, K.-H.: 1971, this volume, p. 770.

Kuklin, G. V.: 1971, this volume, p. 737.

Leighton, R. B.: 1964, Astrophys. J. 140, 1559.

Leighton, R. B.: 1969, Astrophys. J. 156, 1.

Lortz, D.: 1968, Plasma Phys. 10, 967.

Maheswaran, M.: 1969, Monthly Notices Roy. Astron. Soc. 145, 435.

Moffatt, H. K.: 1970, J. Fluid Mech. 41, 435.

Nakagawa, Y.: 1971, this volume, p. 725.

Nakagawa, Y. and Swartztrauber, P.: 1969, Astrophys. J. 155, 295.

Parker, E. N.: 1955, Astrophys. J. 122, 293.

Parker, E. N.: 1963, Astrophys. J. 138, 552.

Parker, E. N. 1970a, Astrophys. J. 160, 383.

Parker, E. N.: 1970b, Ann. Rev. Astron. Astrophys. 8, 1.

Plaskett, H. H.: 1959, Monthly Notices Roy. Astron. Soc. 119, 197.

Plaskett, H. H.: 1962, Monthly Notices Roy. Astron. Soc. 123, 541.

Plaskett, H. H.: 1966, Monthly Notices Roy. Astron. Soc. 131, 407.

Plaskett, H. H.: 1970, Monthly Notices Roy. Astron. Soc. 148, 149.

Roberts, G. O.: 1970, Phil. Trans. Roy. Soc. A 266, 535.

Roberts, G. O.: 1971, to appear.

Roberts, P. H.: 1971, in Lectures in Applied Mathematics (ed. by W. H. Reid), American Mathematical Society, Washington.

Savage, B. D.: 1969, Astrophys. J. 156, 707.

Schmidt, H. U.: 1968, in K. O. Kiepenheuer (ed.), 'Structure and Development of Solar Active Regions', IAU Symp. 35, 95.

Severny, A. B.: 1971, this volume, p. 675.

Simon, G. W. and Weiss, N. O.: 1968, Z. Astrophys. 69, 435.

Starr, V. P.: 1968, Energetics of Eddy Actions in the Solar Atmosphere and Related Astronomical Topics, MIT Dept. of Meteorology, Planetary Circulations Project, Report No. A3.

Starr, V. P. and Gilman, P. A.: 1965, Astrophys. J. 141, 1119.

Steenbeck, M. and Krause, F.: 1966, Z. Naturforsch. 21a, 1285.

Steenbeck, M. and Krause, F.: 1969, Astron. Nachr. $291,49$.

Steenbeck, M., Krause, F., and Rädler, K.-H.: 1966, Z. Naturforsch. 21a, 369.

Sweet, P. A.: 1971, this volume, p. 457.

Ward, F.: 1965, Astrophys. J. 141, 534.

Ward, F.: 1966, Astrophys. J. 145, 416.

Weiss, N. O.: 1964, Phil. Trans. Roy. Soc. A 256, 99.

Weiss, N. O.: 1965, Observatory, 85, 37.

Weiss, N. O.: 1966, Proc. Roy. Soc. A 293, 310.

Weiss, N. O.: 1969, in Plasma Instabilities in Astrophysics (ed. by D. G. Wentzel and D. E. Tidman), Gordon and Breach, New York.

Wilcox, J. M. and Howard, R.: 1970, Solar Phys. 13, 251.

Wilcox, J. M. and Ness, N. F.: 1967, Solar Phys. 1, 437.

Wilson, P. R.: 1969, Solar Phys. 10, 404.

Wilson, P. R.: 1971, this volume, p. 475. 


\section{Discussion}

Sreenivasan: It is very nice to hear that you have obtained magnetic energy density an order of magnitude higher than kinetic energy density in your computations.

(1) Did you actually solve the Navier-Stokes equation with a $(\mathbf{j} \times \mathbf{B})$ term on the right hand side and integrate

$$
\frac{\partial \mathbf{B}}{\partial t}=\nabla \times(\mathbf{u} \times \mathbf{B})+\lambda \nabla^{2} \mathbf{B}
$$

to obtain your flux concentration at the edges of the cells?

(2) If the answer to question (1) is yes, how does one physically understand your conclusion, in the light of equipartition arguments of Batchelor?

(3) What is the reaction due to the growing magnetic field doing to the velocity field?

I have recently shown that, starting from the assumption that an initial magnetic field is force-free, there is only one class of velocity fields which permit the magnetic field to remain force-free in time. These are Beltrami fields and obey the relation:

$$
\boldsymbol{\xi}=\boldsymbol{\alpha} \boldsymbol{\xi} \text { and } \boldsymbol{\nabla} \times \boldsymbol{\eta}=\alpha \boldsymbol{\eta}
$$

where $\boldsymbol{\xi}=(\mathbf{B} \cdot \nabla) \mathbf{u}$ and $\boldsymbol{\eta}=(\mathbf{u} \cdot \nabla) \mathbf{B}$ and $\mathbf{B}$ is given by $\nabla \times \mathbf{B}=\alpha \mathbf{B}$ for incompressible flow. The compressible case is more complicated. It has also been shown that these helial motions can amplify the magnetic field $\mathbf{B}$, beyond the equipartition limit, provided $\alpha$ satisfies an inequality. In this picture, there is no reaction by the magnetic field since the Lorentz force is zero. The amplification limit is set by the dynamical stability of the configuration.

Weiss: Yes, the time-dependent Navier-Stokes, induction and heat flow equations were solved simultaneously. Of course, the enhanced magnetic field tends to slow down the motion, particularly where B is strongest. But the magnetic field is only concentrated locally, at the boundaries of a cell, so the overall energy density, averaged over a whole cell, is not necessarily larger than the average kinetic energy density. Moreover, equipartition arguments, which relate magnetic fields to velocities or vorticity, apply to three dimensional homogeneous turbulence and the numerical experiments don't really reproduce this ideal configuration.

Gilman: As far as the observational evidence from sunspot motion is concerned (in particular the correlation of longitude and latitude motion giving an equatorward transport of momentum) we can not tell the difference between a giant convective cell and a baroclinic or Rossby wave. 